OPEN

EXPERT CONSENSUS DOCUMENT

\title{
A ‘diamond' approach to personalized treatment of angina
}

Roberto Ferrari ${ }^{1,2}$, Paolo G. Camici ${ }^{3}$, Filippo Crea ${ }^{4}$, Nicolas Danchin ${ }^{5}$, Kim Fox 6 , Aldo P. Maggioni ${ }^{7}$, Athanasios J. Manolis ${ }^{8}$, Mario Marzilli9, 10, Giuseppe M. C. Rosano ${ }^{11,12}$ and José L. Lopez-Sendon ${ }^{13}$

Abstract | In clinical guidelines, drugs for symptomatic angina are classified as being first choice ( $\beta$-blockers, calcium-channel blockers, short-acting nitrates) or second choice (ivabradine, nicorandil, ranolazine, trimetazidine), with the recommendation to reserve second-choice medications for patients who have contraindications to first-choice agents, do not tolerate them, or remain symptomatic. No direct comparisons between first-choice and second-choice treatments have demonstrated the superiority of one group of drugs over the other. Meta-analyses show that all antianginal drugs have similar efficacy in reducing symptoms, but provide no evidence for improvement in survival. The newer, second-choice drugs have more evidence-based clinical data that are more contemporary than is available for traditional first-choice drugs. Considering some drugs, but not others, to be first choice is, therefore, difficult. Moreover, double or triple therapy is often needed to control angina. Patients with angina can have several comorbidities, and symptoms can result from various underlying pathophysiologies. Some agents, in addition to having antianginal effects, have properties that could be useful depending on the comorbidities present and the mechanisms of angina, but the guidelines do not provide recommendations on the optimal combinations of drugs. In this Consensus Statement, we propose an individualized approach to angina treatment, which takes into consideration the patient, their comorbidities, and the underlying mechanism of disease.

Correspondence to R.F. Centro Cardiologico Universitario, University Hospital of Ferrara, Via Aldo Moro 8, 44124 Cona, Ferrara, Italy. fri@unife.it
Chronic stable angina pectoris is the most prevalent symptomatic manifestation of ischaemic heart disease, and its management is a priority (BOX 1). Current clinical guidelines recommend antianginal therapy to control symptoms, before considering coronary artery revascularization ${ }^{1-4}$. However, revascularization by either percutaneous coronary angioplasty or CABG surgery is indicated in patients who have significant artery stenosis (50\% left main narrowing or proximal three-vessel disease) to reduce myocardial ischaemia and its adverse clinical manifestation. Antianginal agents are approved by documenting that they improve total exercise duration, together with a reduction in daily frequency of chronic stable angina compared with placebo and/or equivalence to an active comparator. Cardiovascular outcomes, although highly advocated, are not a prerequisite for regulatory approval. None of the antianginal drugs has been proved to reduce cardiovascular mortality or the rate of myocardial infarction. When patients are optimally treated, mortality for chronic stable angina is low, which might explain why all trials designed to improve prognosis have been negative. Guidelines recommend a first-choice and a second-choice approach, based more on tradition and expert opinion, rather than evidence. This categorical approach has been questioned in the past couple of years ${ }^{5-8}$. Newer antianginal drugs, which are classified as second choice, have more evidence-based clinical data that are more contemporary to support their use than is available for the traditional first-choice drugs. Equally, the often-needed combination of double or triple therapy is based on expert opinion and not related to the underlying pathophysiology. What constitutes optimal antianginal treatment, therefore, varies considerably between countries, and the majority of doctors treat their patients according to their own preconceptions.

A group of experts with experience and interest in chronic stable angina met at the University of Ferrara, Italy, to discuss an individualized approach to medical treatment of chronic stable angina, on the basis 


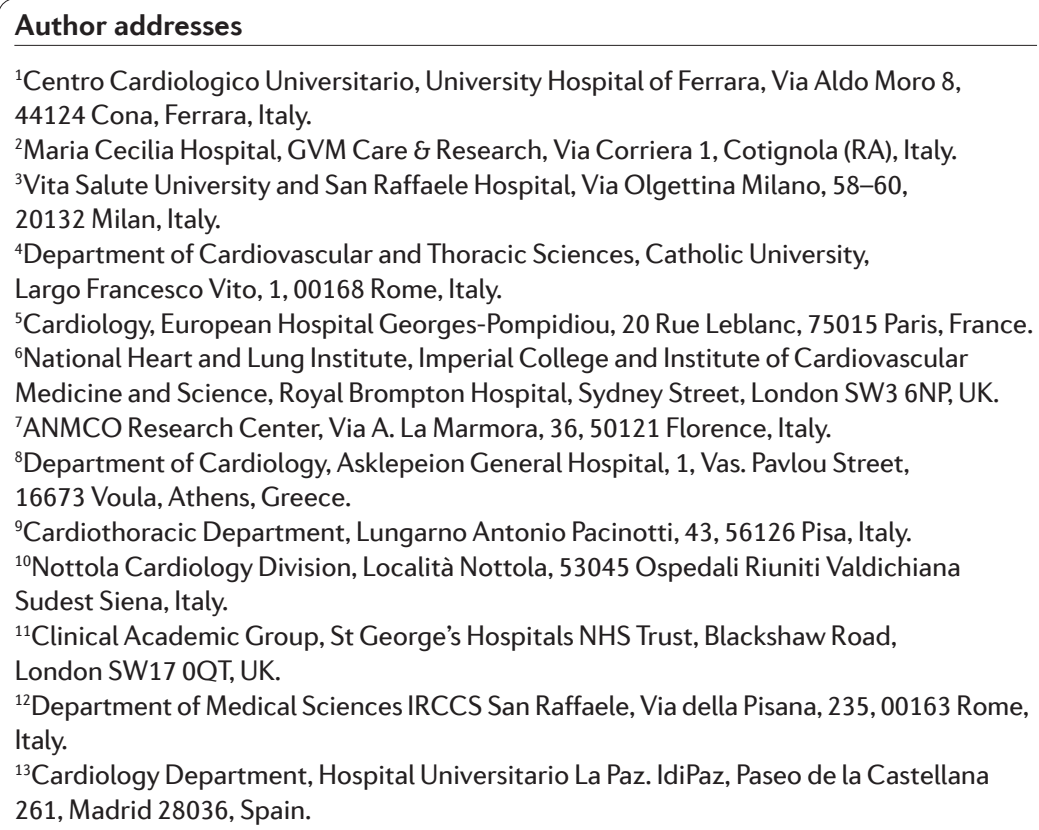

of the underlying physiopathology and comorbidities. We reached the consensus that a 'diamond' approach is more appropriate than current recommendations to guide clinicians in selecting the most suitable drug regimen, alone or in combination, for an individual patient. Thus, the diamond diagram (FIG. 1) has become a theme of this Consensus Statement. As in hypertension, the idea of a diamond approach leaves treating physicians free to choose the most appropriate drugs, according to a patient's needs.

\section{Pathophysiology}

Various pathophysiological entities can cause myocardial ischaemia and, therefore, its symptom: chronic stable angina (FIG. 2). Chronic stable angina can be divided into three major categories: stable, vasospastic, and microvascular.

Chronic stable angina is precipitated by exerciseinduced or emotional stress-induced ischaemia in patients with coronary flow-limiting atherosclerotic stenosis in the large coronary arteries. Several factors contribute to an increase in myocardial oxygen demand, the most important being changes in heart rate, blood pressure or afterload, myocardial wall tension, cardiac hypertrophy, and myocardial contractility. Conversely, the major determinants of oxygen delivery include coronary blood flow, which, in turn, depends on the pressure gradient across the coronary circuit and the integrity of the coronary arteries, as well as on the oxygen-carrying capacity of the blood and the haemoglobin level.

The heart is extremely dependent on adequate oxygen delivery to allow the physiological production of energy (in the form of ATP) to support its contraction and basal metabolism. In 1 day, the human heart performs about 100,000 beats, pumps about 9,0001 of blood around the circulation, and uses a considerable amount of ATP (estimated at $16-30 \mathrm{~kg}$ ), which is produced by the mitochondrial oxidation of acetyl-CoA and immediately utilized to allow contraction of the myofilaments in systole and calcium extrusion in diastole. This energy usage explains the high number of mitochondria in the heart, the high capacity (70-80\%) of the myocytes to extract oxygen from the arterial blood, and why the heart depends on oxygen delivery through the coronary arteries. Under physiological conditions, an increased oxygen demand is met by an increase in coronary blood flow as a consequence of dilatation of coronary arteries, which does not occur in patients with atherosclerotic lesions of epicardial coronary arteries.

The concept that chronic stable angina is caused by epicardial stenosis has been challenged on the basis of various observations. In a fraction of patients, myocardial ischaemia can persist or reoccur after having undergone successful complete coronary revascularization $^{9,10}$. Outcome analyses show little or no prognostic effect of elective angioplasty ${ }^{11}$. Autopsy studies of patients with chronic stable angina suggest that coronary artery obstruction is not necessarily synonymous with myocardial ischaemia ${ }^{12}$. Therefore, it seems that myocardial ischaemia can occur in the absence of obstructive epicardial and coronary atherosclerosis. In the majority of these cases, chronic stable angina is caused by coronary microvascular dysfunction. This condition, previously known as cardiac syndrome $\mathrm{X}$, is named microvascular angina, which often pertains to women with symptoms of chronic stable angina, normal or near-normal coronary arteries, and evidence of ischaemia during stress testing or during acetylcholine testing ${ }^{13,14}$. Increasing attention has been focused on the recognition of this condition, and microvascular angina might occur in up to $40 \%$ of patients with angina, depending on the studies ${ }^{15-17}$. This high percentage, however, includes patients with the symptoms of chronic stable angina with suspected ischaemia, but without a clear demonstration of it.

Another circumstance in which the coronary arteries might appear normal under coronary angiography is with vasospastic angina. This condition occurs in a minority of patients, and is caused by an abnormal reactivity of the smooth muscles of the coronary arteries (which, therefore, cannot be considered functionally normal) ${ }^{18}$. This form of angina presents with specific characteristics - pain is not triggered by exercise, but occurs at rest. Vasospasm can be evoked by different triggers and needs specific diagnostic tests and therapies $^{18}$. A certain degree of coronary spasm might also be superimposed on a nonocclusive, fixed atherosclerotic stenosis, which becomes occlusive and therefore symptomatic when the smooth muscle constricts.

The various causes and mechanisms of chronic stable angina without obstructive coronary artery disease can be grouped into three broad categories: noncardiac, cardiac nonischaemic, and cardiac ischaemic causes (FIG. 3).

\section{Drug therapy}

The management of chronic stable angina encompasses lifestyle modification, control of risk factors for coronary artery disease, and both invasive and noninvasive 
treatment ${ }^{1-4}$. Pharmacological therapy has two main goals: firstly to alleviate symptoms, increase angina-free walking time, and improve quality of life; and secondly to prevent cardiovascular events, mainly myocardial infarction and death. Unfortunately, evidence-based studies indicate that these two goals cannot be achieved with the same class of drugs - pharmacological therapy to prevent cardiovascular events (aspirin, statins, and angiotensin-converting-enzyme inhibitors) does not alleviate symptoms and, similarly, symptomatic therapy does not improve prognosis.

In this Review, we consider only the drug therapies to reduce the symptoms of chronic stable angina (TABLE 1). We summarize the most relevant features of the guideline-recommended drugs, with the aim of emphasizing the different characteristics in terms of mechanism of action, adverse effects, and peculiarities that allow treatment to be targeted to individual patients. A combination of two or more of anti-ischaemic drugs with additive or synergistic effects is often needed to control symptoms effectively. These combinations should also be tailored to the type of chronic stable angina and to the individual patient (FIG. 1).

Nitrates. Nitrates are organic products that, in the vessel wall, undergo enzymatic denitrification by mitochondrial aldehyde dehydrogenase, producing vasoactive nitric oxide, which causes vasodilatation ${ }^{19}$. At low doses, nitrates are predominantly venodilators and thus reduce preload and venous return to the heart with reductions in ventricular volume and myocardial wall tension, leading to a decrease in myocardial oxygen demand. At higher doses, nitrates also dilate coronary arteries, prevent coronary spasm, improve subendocardial blood flow to the ischaemic areas, decrease ventricular diastolic pressure, and lower blood pressure ${ }^{20}$. In addition, nitrates improve collateral blood flow. The effects on preload and afterload are often partly offset by an increase in heart rate and myocardial contractility owing to reflex sympathetic activity. Combining nitrates with $\beta$-blockers can be useful to block tachycardia, leading to a synergetic anti-ischaemic effect ${ }^{1,2}$ (FIG. 1).

\section{Box 1 | Epidemiology and prognosis of chronic stable angina}

- The prevalence and incidence of chronic stable angina vary according to the diagnostic criteria used

- In Western countries, an estimated 30,000-40,000 people per million of the population have chronic stable angina ${ }^{98}$

- The prevalence increases with age in both sexes: estimates range from $5-7 \%$ in women aged $45-64$ years to $10-12 \%$ in women aged $65-84$ years; and from $4-7 \%$ in men aged $45-64$ years to $14-15 \%$ in men aged $65-84$ years $^{1}$

- The incidence of chronic stable angina is $1 \%$ for Western male population aged 45-65 years, with a slightly higher incidence in women ${ }^{1}$

- The prevalence and incidence of chronic stable angina is anticipated to increase in the coming decade as a result of the ageing population, the epidemic of obesity and other risk factors, the increasing use of life-prolonging therapies, and the improved management of acute coronary syndromes ${ }^{99}$

- Approximately 4.1 million deaths from coronary artery disease occur in Europe each year, with $82 \%$ in people aged $>65$ years, and $46 \%$ in those aged $>75$ years; the annual death rate is $1.2-2.4 \% \%^{1,2}$
The most important adverse effects of nitrates are headache, flushing, palpitations, and hypotension. These effects are caused by vasodilatation of intracranial and extracranial vessels, leading to reflex tachycardia. Rare cases of syncope might occur in the elderly owing to profound hypotension associated with bradycardia. All these adverse effects are dose-dependent. Consequently, nitrates should be titrated to control chronic stable angina at the lowest possible dose. Nitrates are contraindicated in patients with hypertrophic obstructive cardiomyopathy, severe aortic or mitral stenosis, or constrictive pericarditis, because they reduce venous return, increase outflow gradient, and reduce cardiac output, and are also contraindicated in patients with closed-angle glaucoma ${ }^{21}$. Concomitant administration of phosphodiesterase 5 inhibitors (used for the treatment of erectile dysfunction or pulmonary hypertension) greatly potentiates the vasodilator effect of nitrates.

\section{Key points for nitrates:}

- Several meta-analyses showed no differences in symptom relief or exercise-testing parameters between nitrates and other antianginal agents ${ }^{22-24}$.

- No data are available to support the concept that nitrates improve outcomes in patients with chronic stable angina.

- The vasodilatory effects of nitrates are independent from the integrity of the endothelium as they undergo bioconversion into nitric oxide both in the endothelial cells of the vessel and in the myocytes, by a mechanism that is still not fully elucidated. This finding could be relevant in patients with microvascular angina, which is characterized by dysfunction of the endothelium, but when tested in these subgroups of patients, the results have been disappointing ${ }^{25}$. Furthermore, small arterioles are nitrate-resistant, which explains why nitrates have no action in the microcirculation and evoke less reflex tachycardia than nitroprusside, a direct nitric oxide donor ${ }^{26}$.

- Patients with microvascular or macrovascular angina might also have an overlapping spasm. In these patients, nitrates provide a clear benefit in terms of reduction of symptoms.

- In patients with chronic stable angina, long-term nitrates should be used with caution, together with other vasodilators.

$\boldsymbol{\beta}$-Blockers. $\beta$-Blockers bind to G-proteins, which are linked to $\beta$-adrenergic receptors, thus inhibiting the effects of adrenaline and noradrenaline on these receptors ${ }^{27}$. In patients with chronic stable angina, $\beta$-blockade reduces ischaemic burden by lowering heart rate and myocardial contractility, especially during exercise, as well as by preventing the exercise-induced increase in blood pressure. These changes result in a reduction in oxygen demand at rest and during exercise. Slowing heart rate leads to increased diastolic time and coronary perfusion, thus improving oxygen supply ${ }^{27}$. Other features of $\beta$-blockers include a reduction in atrioventricular conduction and ectopic activity, as well as an attenuation of the release of renin from the juxtaglomerular cells, 


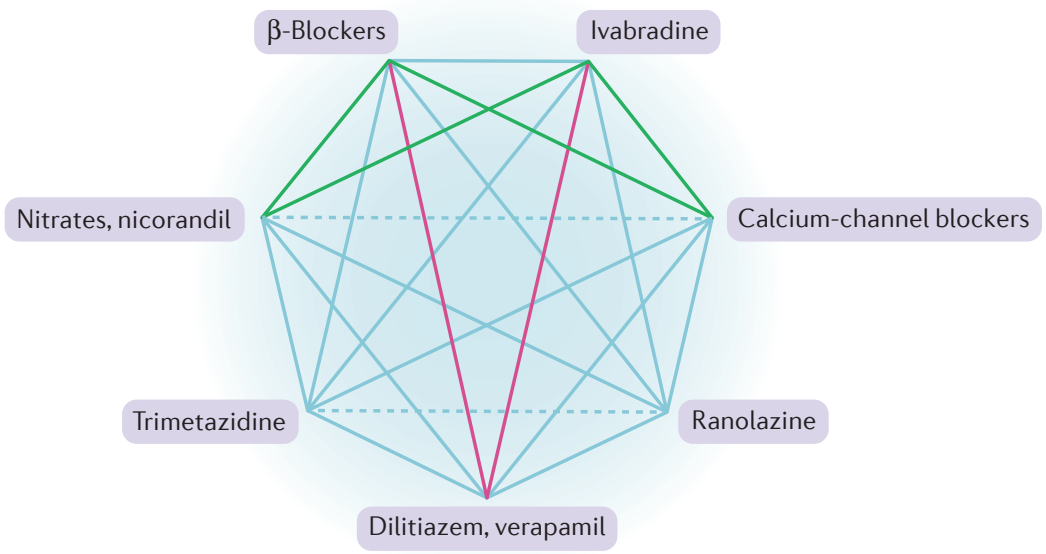

Figure 1 | Possible combinations of different classes of antianginal drugs. The schematic shows useful combinations (green lines), combinations that are not recommended (red lines), possible combinations (blue solid lines), and drugs with similar actions (blue dashed lines). and hypotension, but can be combined with ivabradine to reduce heart rate further and to improve exercise duration ${ }^{29}$.

- $\beta$-Blockers should be the preferred treatment in patients with reduced left ventricular ejection fraction $(<40 \%)$ or with post-infarct angina, or heart failure. Several randomized clinical trials in this subset of patients have shown an improvement in angina associated with a reduction in cardiovascular mortality and sudden death ${ }^{30,31}$.

- $\beta$-Blockers should be the first-choice treatment in patients with chronic stable angina and high blood pressure and heart rate, history of supraventricular tachycardia including atrial fibrillation, or hypertrophic cardiomyopathy. These suggestions are reasonable and are made by experts, but no specific trials have been carried out in these populations.

- Traditionally, $\beta$-blockers are considered to be cardioprotective in patients with stable coronary artery disease, independently from left ventricular dysfunction. This assumption is not supported by evidence, and is extrapolated from data obtained in the early 1980s in patients after myocardial infarction, in the era before revascularization and in the absence of treatment with angiotensin-converting-enzyme inhibitors and statins $^{32}$. Clinical and observational research shows that the beneficial prognostic effect of heart-rate-lowering drugs, such as $\beta$-blockers (and ivabradine), depends on the effects of reduced heart rate on the damaged myocytes, with improvement of ventricular remodelling ${ }^{33}$. In the majority of patients with chronic stable angina, ventricular function is preserved, with no remodelling process. In these patients, $\beta$-blockers do not improve outcome, as shown in contemporary observational studies $^{31,33-35}$. Accordingly, the guidelines have, over the years, limited the prognostic usefulness of $\beta$-blockade in patients with chronic stable angina.

- Nonselective, and also to some extent selective, $\beta$-blockers can result in coronary constriction by causing a shift from $\beta$-adrenoreceptors to $\alpha$-adrenoreceptors. Under these circumstances, catecholamines bind preferentially to the $a$-adrenoreceptors causing vasoconstriction. $\beta$-Blockers are contraindicated in vasospastic angina, and not useful in microvascular angina.

Calcium-channel blockers. Calcium-channel blockers are classified according to their structure as being dihydropyridines (amlodipine, felodipine, nifedipine, and nisoldipine) or nondihydropyridines (diltiazem and verapamil). All these drugs inhibit calcium influx through the high-voltage-activated L-type calcium channel, located in the smooth muscle of the arterial wall and on the myocardium, leading to smooth muscle relaxation and reduction of myocardial contractility. The different agents have different selectivity for L-type calcium channels in the vasculature and myocardium ${ }^{27}$. Dihydropyridines have high selectivity for L-type channels of the vasculature and produce a decrease in coronary and systemic vascular resistance. They exert an antianginal action by reducing oxygen demand and 


\section{CONSENSUS STATEMENT}

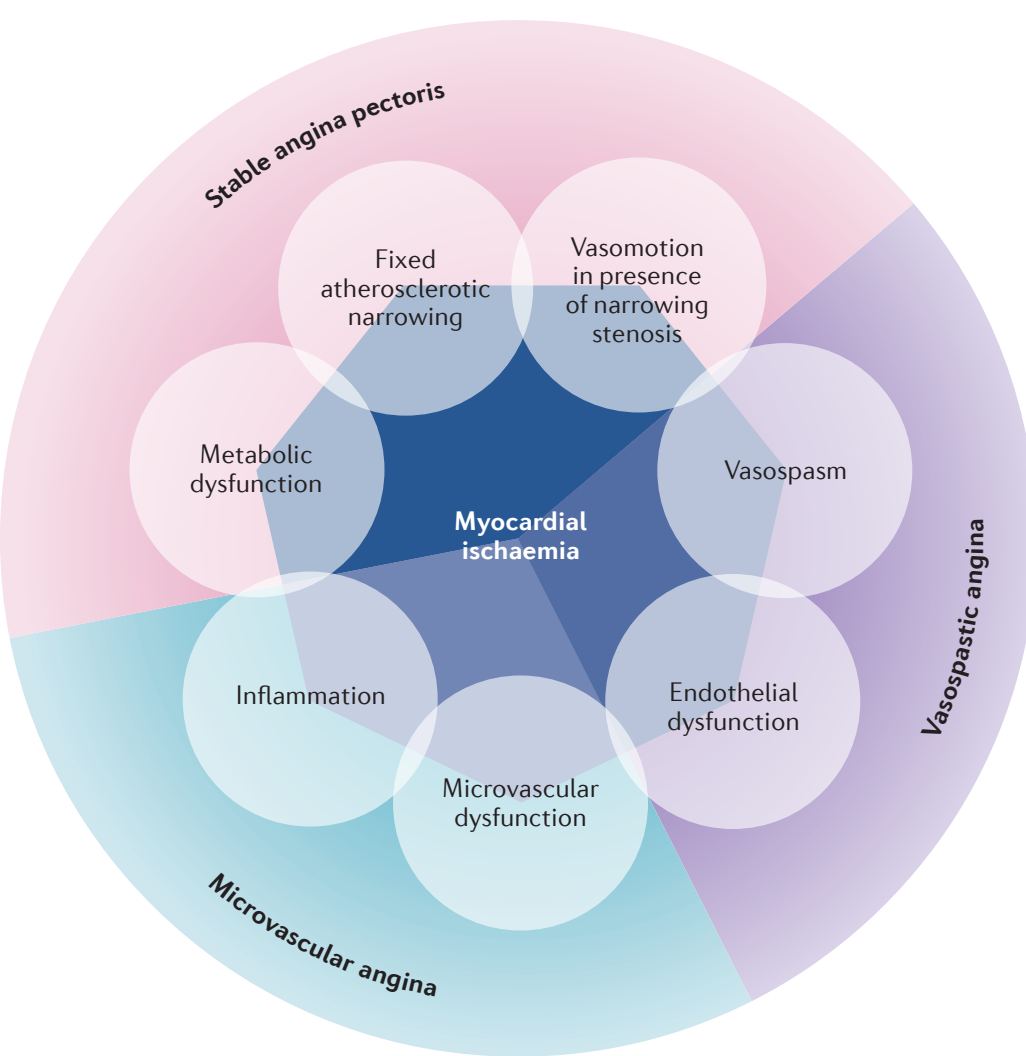

Figure 2 | Different manifestations of myocardial ischaemia. Stable angina occurs when myocardial ischaemia is caused by fixed atherosclerotic narrowing of one or more epicardial coronary arteries. In some circumstances, the angina is associated with a coronary spasm and metabolic dysfunction. Vasospastic angina occurs when myocardial ischaemia is caused by a coronary artery spasm with or without endothelial dysfunction. Microvascular angina refers to the absence of an obstructed epicardial coronary artery. Myocardial ischaemia in this case can be caused by microvascular and/or endothelial dysfunction and inflammation.
- Calcium-channel blockers do not improve survival of patients with chronic stable angina with or without myocardial infarction or left ventricular dysfunction and, when used in patients with heart failure, they might have deleterious effects on outcome ${ }^{37,38}$.

- All calcium-channel blockers are metabolized in the liver by cytochrome P450 3A4, the same enzyme that metabolizes ivabradine. Diltiazem and verapamil should, therefore, not be used in combination with ivabradine owing to the risk of severe bradycardia ${ }^{39}$.

Nicorandil. Nicorandil is a nicotinamide nitrate ester and an adenosine-sensitive potassium-channel opener with nitric oxide donor capacity ${ }^{40}$. Nicorandil exerts an antianginal effect by increasing coronary blood flow, preventing coronary artery spasms, and causing arterial vasodilatation. Therefore, as with nitrates and dihydropyridine calcium-channel blockers, nicorandil improves the balance of oxygen demand and delivery. Additionally, as a consequence of its capacity to open ATP-sensitive potassium channels, nicorandil exerts some metabolic effects and a form of preconditioning on the myocardium $^{41}$. The antianginal action has been assessed in small and dated randomized trials with mixed results ${ }^{41-43}$. The main adverse effects of nicorandil are headache, reflux, tachycardia, facial flushing, and hypotension. Rarely, serious skin, mucosal, and eye ulceration have also been reported ${ }^{43}$.

\section{Key points for nicorandil:}

- Nicorandil is associated with some improvements in outcomes. In the randomized, controlled IONA outcome study ${ }^{44}$, nicorandil significantly reduced the composite primary end point of cardiovascular death, nonfatal myocardial infarction, or unplanned admission to hospital for chest pain, despite $39 \%$ of patients in the treatment group withdrawing because of headache. Of note, the IONA trial ${ }^{44}$ showed no significant reduction in the two most important components of the primary end point: cardiovascular mortality and myocardial infarction. The beneficial effects were driven by a reduction in unplanned hospitalization for unstable angina, which is an indication of reduced angina although, unexpectedly, nicorandil did not improve chronic stable angina in the study. A larger beneficial effect of nicorandil was recorded in a propensity-matched cohort of 5,115 Japanese patients with chronic stable angina who were followed up for 7 years.

- The concomitant use of nicorandil with aspirin might increase the risk of gastrointestinal ulcers, perforations, and haemorrhage ${ }^{43}$.

Ivabradine. By inhibiting the $I_{\mathrm{f}}$ current, ivabradine reduces the slope of the slow diastolic depolarization phase of the action potential in sinus node cells, thereby selectively reducing heart rate. The $I_{\mathrm{f}}$ inhibition is 'usedependent', and the heart-rate reduction with ivabradine is greater when initial heart rate is high, and lower when the initial heart rate is $\operatorname{low}^{45}$. Ivabradine has no negative inotropic or lusitropic effect ${ }^{45}$. The ivabradine-induced 
reduction in heart rate is similar to that with $\beta$-blockers, but with maintenance of coronary dilatation during exercise $^{46}$. In addition, ivabradine increases coronary flow reserve and collateral perfusion, promoting the development of coronary collaterals and maintaining endothelial function in patients with chronic stable angina ${ }^{47,48}$. The antianginal effects of ivabradine have been documented in several randomized clinical trials against placebo, various active comparators, and in addition to $\beta$-blockers, as well as in unselected studies reflecting routine clinical practice ${ }^{29,49}$.

Adverse effects of ivabradine are bradycardia and phosphenes. Occurrence of bradycardia was reported in $2.2-4.2 \%$ of patients with chronic stable angina. The rate of bradycardia was much higher $(18.0 \%)$ in the SIGNIFY trial ${ }^{39}$. Of note, the therapeutic regimen in SIGNIFY (7.5 mg uptitrated to $10.0 \mathrm{mg}$ twice daily) significantly differs from current clinical practice, and $4.6 \%$ of patients were treated with diltiazem or verapamil, which are moderate inhibitors of cytochrome P450 P3A4,

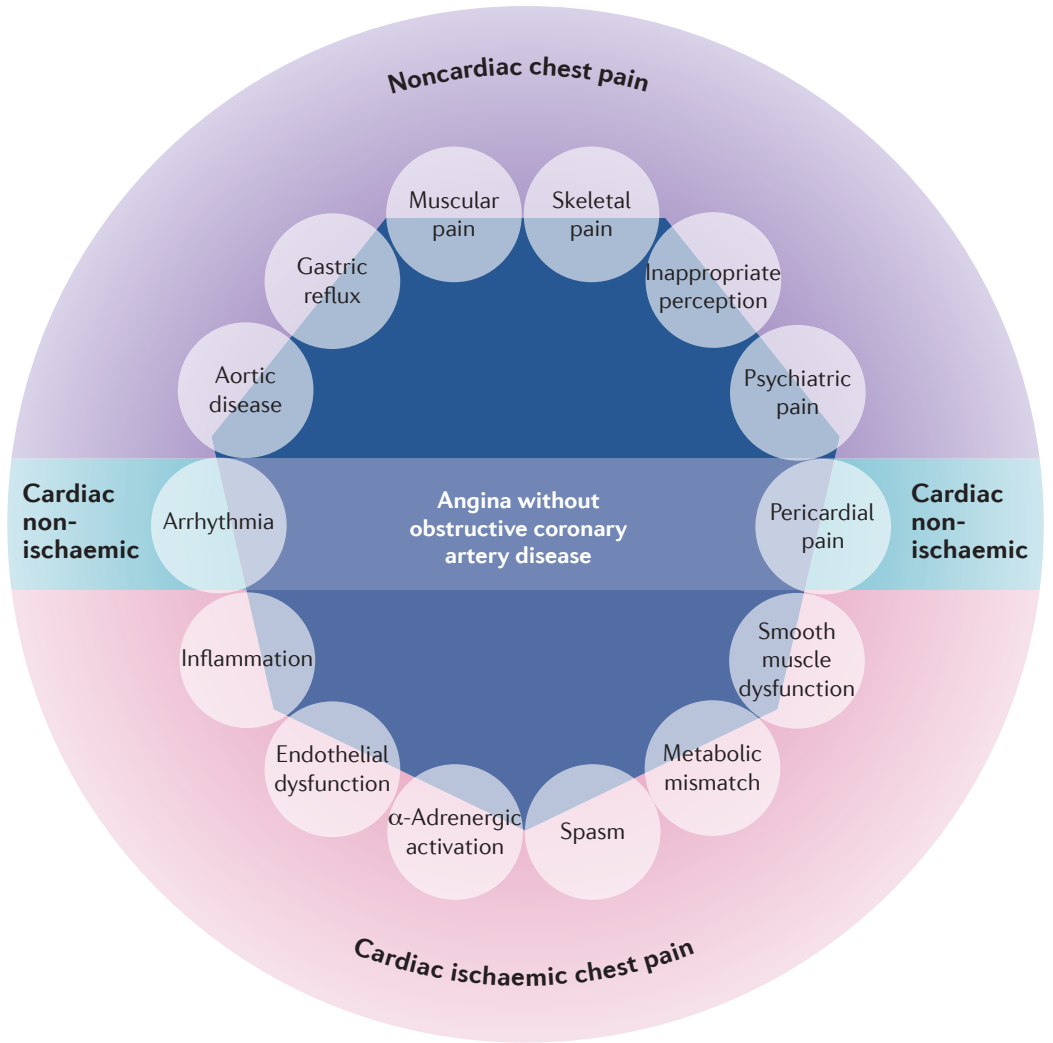

Figure 3 | Aetiology of chest pain without obstructive coronary artery disease.

Various causes of angina without obstructive coronary artery disease are shown. Noncardiac pain can be caused by: gastro-oesophageal reflux, musculoskeletal alterations, pulmonary and aortic diseases, psychiatric conditions, or inappropriate perception of chest pain. Causes of nonischaemic cardiac pain are: pericarditis, pericardial diseases, and arrhythmias. As for the cardiac ischaemic group, spasm of epicardial coronary arteries owing to smooth muscle dysfunction was recognized as an adjunctive, functional mechanism of transient myocardial ischaemia and angina. Heightened sensitivity of the coronary microcirculation to vasoconstrictor stimuli associated with a limited microvascular vasodilator capacity might also be the cause of microvascular angina, together with mismatch between metabolic signalling and microvascular adaptation. Other causes include dysfunction of endothelial cells of the microvascular network, most likely owing to increased oxidative stress and inflammation. which metabolizes ivabradine. This inhibition might have contributed to the excess bradycardia. In SHIFT $^{50}$, involving 6,558 patients with chronic heart failure, despite the frailty of the patients and that $89 \%$ were taking $\beta$-blockers, treatment withdrawal owing to bradycardia occurred in only $1 \%$ of the overall population.

The visual symptoms induced by ivabradine are caused by the presence of $I_{\mathrm{f}}$ channels in the retina that closely resemble the $I_{\mathrm{f}}$ channels of the sinus node. The observed phosphenes are reported to occur in $5.4 \%$ of patients, are mild and transitory, and only rarely lead to treatment withdrawal $(<1 \%)^{39}$.

Treatment with ivabradine also seems to increase the absolute incidence of atrial fibrillation by $0.7 \%{ }^{51}$. The majority of cases of emergent atrial fibrillation in the SIGNIFY trial ${ }^{39}$ were paroxysmal in nature and managed according to current European guidelines.

\section{Key points for ivabradine:}

- The antianginal efficacy of ivabradine is similar to that of other classes of antianginal agents ${ }^{52,53}$.

- Ivabradine provides additional benefits when used in combination with the other antianginal drugs (except diltiazem and verapamil), including $\beta$-blockers ${ }^{29}$.

- The synergistic effect between $\beta$-blockers and ivabradine suggests that in patients receiving treatment with $\beta$-blockers who are still symptomatic, adding ivabradine is more efficient than uptitration of $\beta$-blockers ${ }^{54}$.

- The SIGNIFY trial ${ }^{39}$ data confirm that ivabradine improves symptoms of chronic stable angina, but does not affect outcomes in patients with preserved left ventricular function. In the prespecified chronic stable angina subgroup (Canadian Cardiovascular Society class $\geq 2 ; 12,000$ patients), an increase seemed to occur in the primary composite end point of cardiovascular death and nonfatal myocardial infarction. Of note, however, this subgroup analysis of a neutral trial should be interpreted with caution. In any case, the explanation of these results could be the higher dosage used in the trial and/or the concomitant use of diltiazem or verapamil. The Pharmacovigilance Risk Assessment Committee of the European Medicines Agency concluded after revision of the SIGNIFY data that, at an appropriate dose (up to $7.5 \mathrm{mg}$ twice daily), ivabradine is a useful therapeutic option to relieve symptoms and improve quality of life in suitable patients with chronic stable angina in sinus rhythm with a heart rate $\geq 70 \mathrm{bpm}$ who cannot tolerate or whose symptoms are inadequately controlled with $\beta$-blockers ${ }^{55}$.

- The BEAUTIFUL trial ${ }^{56,57}$ assessed the effects of ivabradine on clinical outcomes. The primary end point (combination of cardiovascular death and hospitalization for myocardial infarction or for new-onset or worsening heart failure) was not improved by ivabradine. In a prespecified subgroup analysis of patients with chronic stable angina at entry, ivabradine reduced the rate of myocardial infarction ${ }^{58}$. These data need to be considered with great caution, as they are the results of a subgroup analysis of a neutral trial. 
Table 1 | Drugs for angina: pharmacology, symptom relief, outcomes benefits, and guideline recommendations

\begin{tabular}{|c|c|c|c|c|c|c|c|c|c|c|}
\hline Antianginal drug & HR & SBP & DBP & PVR & $\mathrm{CC}$ & CV & Symptom relief & Outcomes benefit & ESC* & $\mathrm{ACC} / \mathrm{AHA}^{*}$ \\
\hline \multicolumn{11}{|l|}{ Nitrates } \\
\hline Short-acting & $\uparrow-$ & $\downarrow \downarrow$ & $\downarrow \downarrow$ & $\downarrow-$ & - & $\uparrow \uparrow \uparrow$ & Yes & No & IB & IB \\
\hline \multicolumn{11}{|l|}{$\beta$-Blockers } \\
\hline Noncardioselective & $\downarrow \downarrow \downarrow$ & $\downarrow \downarrow$ & $\downarrow \downarrow$ & $\uparrow-$ & $\downarrow \downarrow$ & - & Yes & No & IA & IB \\
\hline Cardioselective (reduced EF) & $\downarrow \downarrow \downarrow$ & $\downarrow \downarrow$ & $\downarrow \downarrow$ & - & $\downarrow \downarrow$ & - & Yes & Yes & IB & IB \\
\hline With vasodilatation (preserved EF) & $\downarrow \downarrow$ & $\downarrow \downarrow \downarrow$ & $\downarrow \downarrow \downarrow$ & $\downarrow \downarrow$ & $\downarrow$ & - & Yes & No & IB & IB \\
\hline With vasodilatation (reduced EF) & $\downarrow \downarrow$ & $\downarrow \downarrow \downarrow$ & $\downarrow \downarrow \downarrow$ & $\downarrow \downarrow$ & $\downarrow$ & - & Yes & Yes & IA & IA \\
\hline \multicolumn{11}{|l|}{ Calcium-channel blockers } \\
\hline Dihydropyridines & $\uparrow-$ & $\downarrow \downarrow \downarrow$ & $\downarrow \downarrow \downarrow$ & $\downarrow \downarrow \downarrow$ & $\uparrow-$ & $\uparrow \uparrow \uparrow$ & Yes & No & IA & IB \\
\hline Nicorandil & $\uparrow$ & $\downarrow \downarrow$ & $\downarrow \downarrow$ & $\downarrow-$ & - & $\uparrow \uparrow \uparrow$ & Yes & Yes & $\|_{a} B$ & NA \\
\hline Ranolazine & - & - & - & - & - & - & Yes & No & $I_{a} B$ & $\| \mathrm{A}$ \\
\hline Trimetazidine & - & - & - & - & - & - & Yes & No & $\mathrm{II}_{\mathrm{b}} \mathrm{B}$ & NA \\
\hline
\end{tabular}

*Guideline classification of benefit: class $\mathrm{I}=$ benefit $>>>$ risk; class $\|_{\mathrm{a}}=$ benefit $>>$ risk; class $\|_{\mathrm{b}}=$ benefit > risk. Level of evidence: $\mathrm{A}=$ one or two large, randomized trials; $\mathrm{B}$ = one randomized trial or small meta-analysis. CC, cardiac contractility; CV, coronary vasodilatation; DBP, diastolic blood pressure; $\mathrm{EF}$, ejection fraction; $H R$, heart rate; NA, not available; PVR, peripheral vascular resistance; SBP, systolic blood pressure.

- A 2016 analysis of patients with ischaemic heart disease and chronic stable angina enrolled in SHIFT ${ }^{59}$ shows that the outcome benefits of ivabradine were maintained for the duration of follow-up (22.9 months).

Ranolazine. Ranolazine is an active piperazine derivative that is structurally related to trimetazidine ${ }^{60}$. The exact mechanism of action of ranolazine is difficult to elucidate. Firstly, like trimetazidine, ranolazine was shown to promote glucose oxidation, thus improving anaerobic metabolism under ischaemic conditions ${ }^{61}$. Subsequently, ranolazine was shown to cause concentration-dependent, voltage-dependent, and frequency-dependent inhibition of the late sodium current. By inhibiting late sodium efflux, ranolazine prevents intracellular calcium overload and its deleterious effects ${ }^{60}$. The therapeutic concentration needed to reduce calcium overload is similar to that at which an increase in glucose oxidation was observed.

Regardless of the main mechanism of action, ranolazine has been shown to exert an antianginal action, with conflicting results when the immediate-release formulation was used ${ }^{62}$. Reduction of chronic stable angina occurs without affecting heart rate, blood pressure, or myocardial perfusion. Under these circumstances, if one excludes a possible benefit from an improvement in anaerobic metabolism under ischaemic conditions, the only plausible explanation for the antianginal effects is reduced left ventricular wall tension and, therefore, a reduction in oxygen demand owing to prevention of calcium overload during ischaemia ${ }^{60}$. This property also accounts for a prolongation of the corrected QT interval, which leads to contraindication of the drug.
Ranolazine has a good safety profile, being devoid of any adverse haemodynamic effects when used as monotherapy or in combination. Even the dose-related prolongation of the QT interval does not seem to be a concern, at least at the recommended therapeutic doses.

\section{Key points for ranolazine:}

- A systematic review of randomized, controlled trials and a Cochrane meta-analysis concluded that the extended-release formulation of $500 \mathrm{mg}$ or $1,000 \mathrm{mg}$ of ranolazine is effective in reducing symptoms of chronic stable angina, similarly to all the other currently used antianginal agents ${ }^{60}$, and its neutral haemodynamic profile could be useful in cases of bradycardia and/or hypotension.

- Ranolazine does not improve outcomes of patients with chronic stable angina, independently from the ventricular function. The recent RIVER-PCI trial ${ }^{63}$ enrolled patients with chronic stable angina and incomplete revascularization after percutaneous coronary intervention. Ranolazine did not improve the primary end point of death, myocardial infarction, or recurrent chronic stable angina requiring further revascularization. Equally, nonbeneficial effects (major cardiovascular events, including cardiovascular death and myocardial infarction) were found in the MERLIN trial ${ }^{64}$ involving patients with non-ST-segment elevation acute coronary syndrome.

- Ranolazine might be effective in patients with microvascular angina. Following some small, proof-ofconcept studies suggesting a possible benefit, a larger trial was conducted in this specific population 
of patients. The results did not show superiority of ranolazine compared with placebo on the frequency of chronic stable angina or reduction in myocardial ischaemia evaluated by single-photon emission computed tomography ${ }^{65}$.

- Given the effects on the sodium current and on calcium homeostasis, ranolazine might be more effective than other drugs in patients with left ventricular hypertrophy.

- In patients enrolled in the MERLIN trial ${ }^{64}$, ranolazine significantly reduced glycated haemoglobin (HbAlc) levels. Therefore, patients with diabetes and chronic stable angina might benefit from ranolazine treatment.

- Ranolazine is contraindicated in patients with hepatic impairment or liver cirrhosis. These precautions are relevant considering the prolongation of the corrected QT interval. Any accumulation of ranolazine must be avoided, because its effects on QT-interval prolongation are dose-dependent ${ }^{60}$.

- Ranolazine increases digoxin concentration and should be used with caution in patients taking digoxin.

Trimetazidine. Trimetazidine is a piperazine derivative. As with ranolazine, the exact mechanism of the antianginal action of trimetazidine is not fully understood. Trimetazidine increases cellular tolerance to ischaemia by inhibiting the mitochondrial 3-ketoacyl-CoA thiolase and, consequently, by increasing glucose metabolism. In so doing, trimetazidine directs pyruvate into the mitochondria, leading to less proton and lactic acid production from the ischaemic myocardium, and more anaerobic ATP production from the cytosol. The net consequences of these effects are a reduction in free fatty acid oxidation and an increase in glucose utilization by the ischaemic myocardium ${ }^{66}$.

These metabolic effects have been confirmed in the clinical setting, where treatment with trimetazidine for 3 months was shown to increase myocardial levels of high-energy phosphates by $33 \%$ in patients with heart failure $^{67}$. The clinical antianginal effects of trimetazidine have been tested in small and historical studies in patients with chronic ischaemia, both as monotherapy and as part of a combination regimen, with either calcium-channel blockers or $\beta$-blockers ${ }^{68-70}$.

Generally, treatment-related adverse effects are mild and well-tolerated, mostly comprising gastrointestinal disturbances such as nausea, vomiting, and minor headaches. However, trimetazidine is not recommended in patients with Parkinson disease, parkinsonism, and other related movement disorders, or in patients with severe renal impairment (creatinine clearance $<30 \mathrm{ml} / \mathrm{min}$ ).

\section{Key points for trimetazidine:}

- Trimetazidine is a metabolic modulator that does not exert haemodynamic effects. Therefore, unlike all the other first-line and second-line antianginal agents, trimetazidine does not affect oxygen demand, but improves the metabolic efficiency of the ischaemic myocytes.

- In a meta-analysis, trimetazidine significantly improved exercise tolerance, weekly chronic stable angina episodes, and reduced the use of short-acting nitrates in comparison with placebo ${ }^{71}$. The antianginal effects were similar to those of other antianginal agents $^{23}$.

\section{Guideline recommendations}

All guidelines take into consideration evidence provided by published trial data and meta-analysis of the different studies. However, in absence of these data - which unfortunately is not uncommon - the recommendations are made according to previously reported guidelines, tradition-driven beliefs, and opinion of the experts on the guideline committees. Some guideline suggestions are inevitably, therefore, not evidence-based. The lack of reliable, objective data is indicated by providing a lower class to the recommendation.

The absence of objective data is particularly relevant to the guidelines for antianginal drug therapy ${ }^{5-8}$. The $\mathrm{AHA} / \mathrm{ACC}^{2}, \mathrm{ESC}^{1}$, and $\mathrm{NICE}^{4}$ recommendations suggest a first-choice therapy with sublingual or short-acting nitroglycerin, $\beta$-blockers, and calcium-channel blockers. Ivabradine, nicorandil, ranolazine, and trimetazidine are reserved for patients who have contraindications to the first-choice agents, do not tolerate them, or remain symptomatic, even though more evidence-based clinical data that are more contemporary are available for them than for the first-choice drugs.

No head-to-head comparisons between first-choice and second-choice treatments are available that demonstrate superiority of one over any other in terms of antianginal effects. Furthermore, double and sometimes triple therapy with different classes of antianginal drugs is often needed, and the guidelines do not provide an indication of the optimal combination. Nevertheless, the literature and our discussion clearly show that some agents have specific properties (in addition to their antianginal effect) that allow the selection of the best treatment for the specific physiopathology or for the comorbidities and/or risk factors. Our 'diamond' approach is a possible framework for individualized therapy using the established antianginal drugs, which could help clinicians to make the best selection of medications for their patients.

\section{'Diamond' approach to treatment}

Particular drugs or combinations of drugs are preferred for specific physiopathologies or comorbidities (FICS 4,5).

High heart rate. Heart-rate-lowering agents, such as $\beta$-blockers, nondihydropyridine calcium-channel blockers (diltiazem and verapamil), and ivabradine are the preferred drugs when heart rate is $>70 \mathrm{bpm}$. Ivabradine can be safely and usefully added to $\beta$-blockers when heart rate remains elevated $(\geq 70 \mathrm{bpm})^{29}$, but combining ivabradine with diltiazem or verapamil is clearly contraindicated ${ }^{39}$. Vasodilators, such as dihydropyridine calcium-channel blockers and nitrates, are less attractive because they might increase heart rate. The other antianginal drugs can be co-administered, if necessary. Combining $\beta$-blockers with diltiazem or verapamil is, however, not recommended owing to risk of highdegree atrioventricular block. No consensus exists on 
the optimal heart-rate target. The European Medicines Agency recommends that the heart-rate-lowering agents should be administered when heart rate is $>70 \mathrm{bpm}$, but the dose should be decreased if heart rate remains below 50-55 bpm (FIG. 4).

Low heart rate. When heart rate is low ( $\leq 50-55 \mathrm{bpm})$, heart-rate-slowing agents are clearly contraindicated. Preference should be for dihydropyridine calciumchannel blockers and nitrates or nicorandil, because these might increase heart rate by evoking a sympathetic reflex. Other antianginal agents such as ranolazine and trimetazidine can be considered (FIG. 4).

Hypertension. $\beta$-Blockers and dihydropyridine calciumchannel blockers are preferred in hypertension. Blood pressure should not be reduced below $130 \mathrm{mmHg}$ systolic and $80 \mathrm{mmHg}$ diastolic because, in patients with coronary artery disease, a clear J-curve phenomenon has been demonstrated ${ }^{72}$. Even in the presence of diabetes and other risk factors, no reason exists to adopt an aggressive blood-pressure-lowering treatment, as previously suggested. A meta-analysis showed that in patients with diabetes and a baseline systolic blood pressure level $<140 \mathrm{mmHg}$, further blood-pressure lowering resulted in an increased risk of cardiovascular death ${ }^{73}$ (FIG. 4).

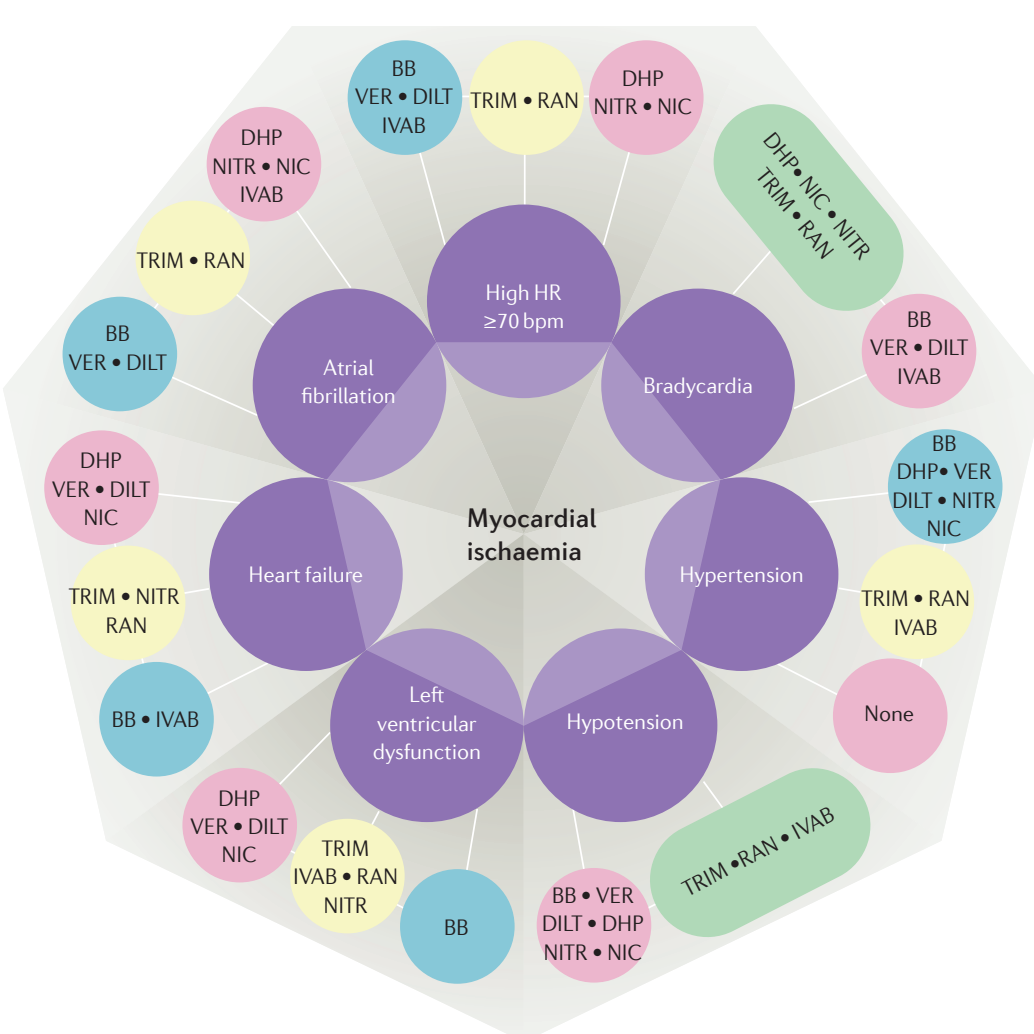

Preferred

All possible

Co-administered

Contraindicated or caution needed

Figure 4 | Possible combinations of classes of antianginal drugs according to different comorbidities. BB, $\beta$-blockers; DHP, dihydropyridine calcium-channel blockers; DILT, diltiazem; HR, heart rate; IVAB, ivabradine; NIC, nicorandil; NITR, nitrates; RAN, ranolazine; TRIM, trimetazidine; VER, verapamil.
Hypotension. Agents such as calcium-channel blockers, nitrates, and $\beta$-blockers that significantly decrease blood pressure should not be used in patients with angina and low blood pressure, because they might impair coronary perfusion. No validated threshold exists for high versus low blood pressure in patients with coronary artery disease. Considering the available data, a threshold of $130 \mathrm{mmHg}$ systolic and $80 \mathrm{mmHg}$ diastolic is reasonable $\mathrm{e}^{72,73}$. In patients with lower blood pressure, the use of ivabradine (if associated with a concomitant increase in heart rate), ranolazine, or trimetazidine is preferable (FIG. 4).

Left ventricular dysfunction and heart failure. When chronic stable angina is present in patients with left ventricular systolic dysfunction with or without overt heart failure, the overwhelming, evidence-based indication is to use $\beta$-blockers, which can reduce chronic stable angina and, at the same time, effectively reduce cardiovascular morbidity and mortality in these patients $s^{30,31}$. These beneficial effects seem to be directly correlated with a heart-rate-lowering effect of $\beta$-blockers; therefore, $\beta$-blockers with intrinsic sympathetic activity should be avoided. If the heart rate remains elevated (>70 bpm), despite optimal $\beta$-blockade, ivabradine should be considered. The SHIFT trial ${ }^{50}$ showed a further prognostic benefit of adding ivabradine to evidence-based optimal therapy in patients with overt heart failure and reduced ejection fraction. Similar benefits were also seen in the subgroup with chronic stable angina ${ }^{59}$. Diltiazem and verapamil should be used with caution in this subset of patients because they can worsen left ventricular dysfunction ${ }^{74}$. Finally, meta-analysis of small studies of patients with left ventricular dysfunction and/or heart failure suggests that trimetazidine might be beneficial in addition to recommended therapy ${ }^{75}$ (FIC. 4).

Atrial fibrillation. Atrial fibrillation might aggravate anginal symptoms owing to increased heart rate. Therefore, agents such as $\beta$-blockers and nondihydropyridine calcium-channel blockers should be preferred when this comorbidity is present. Conversely, because of its selectivity for $I_{\mathrm{f}}$ channels, ivabradine is ineffective in patients with atrial fibrillation, and might even increase the incidence of the arrhythmia. A meta-analysis showed that treatment with ivabradine is associated with a relative risk of atrial fibrillation of 1.15 (REF. 76). The SIGNIFY trial ${ }^{39}$ showed significantly more atrial arrhythmias in patients treated with ivabradine $(5.3 \%)$ versus placebo (3.8\%). For these reasons, ivabradine is contraindicated in patients with chronic stable angina and atrial fibrillation. Dihydropyridine calcium-channel antagonists and nitrates or nicorandil should also be avoided because they can further increase heart rate, whereas the other agents can be added to $\beta$-blockers to control the symptoms of chronic stable angina further. The addition of ranolazine, which has been shown to suppress supraventricular arrhythmias and atrial fibrillation, might be particularly useful $^{77,78}$ (FIG. 4). 


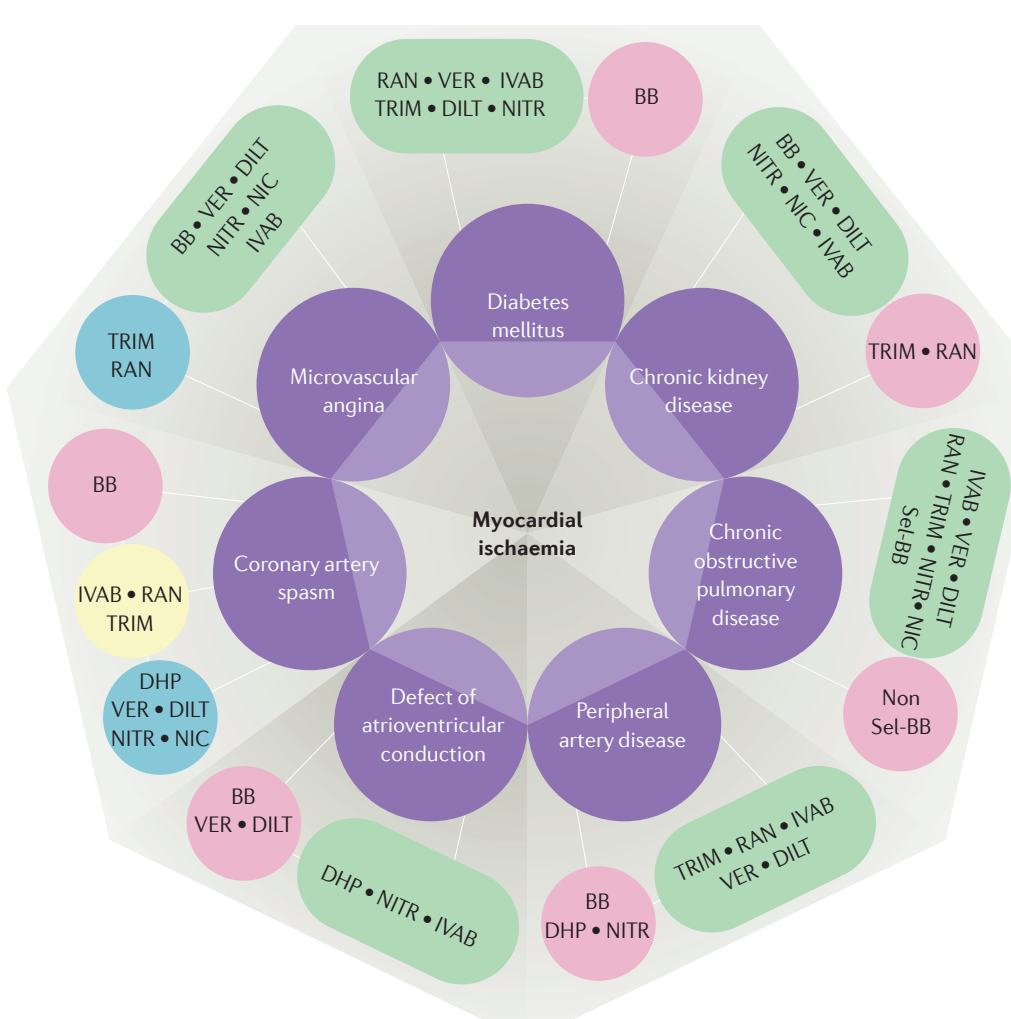

Preferred All possible

Co-administered

Contraindicated or caution needed

Figure 5 | Possible combinations of classes of antianginal drugs according to different comorbidities. BB, $\beta$-blockers; DHP, dihydropyridine calcium-channel blockers; DILT, diltiazem; IVAB, ivabradine; NIC, nicorandil; NITR, nitrates; Non Sel-BB, nonselective $\beta$-blockers; RAN, ranolazine; Sel-BB, $\beta_{1}$-selective blockers; TRIM, trimetazidine; VER, verapamil.
Traditionally, $\beta$-blockers were considered to facilitate new-onset diabetes and aggravate glycaemic control. For these reasons, $\beta$-blockade is avoided in patients with diabetes and chronic stable angina. However, newer vasodilating $\beta$-blockers, such as carvedilol and nebivolol, have been reported to improve insulin sensitivity, overcoming the metabolic limitation of the traditional $\beta$-blockers ${ }^{82}$. All the other antianginal drugs can be used to ameliorate the ischaemic burden (FIG. 5).

Chronic kidney disease. Concomitant chronic kidney disease is a risk factor often associated with coronary artery disease and has a major effect on outcome and therapeutic decision-making. Unfortunately, this group of patients is poorly explored in clinical trials because chronic kidney disease is normally an exclusion criterion. Evidence is, therefore, lacking. Ranolazine and trimetazidine should not be prescribed when glomerular filtration rate is $<30 \mathrm{ml} / \mathrm{min} / 1.73 \mathrm{~m}^{2}$. Ranolazine undergoes extensive hepatic metabolism by cytochrome $\mathrm{P} 450$ and is primarily excreted by the kidney. Plasma levels increase up to $50-60 \%$ in patients with moderate hepatic or renal impairment ${ }^{83}$. Not surprisingly, given that they belong to the same pharmacological class, the limitations of ranolazine also exist for trimetazidine. Therefore, these drugs should not be used in patients with chronic stable angina and chronic kidney disease, because they carry the risk of further complications. No contraindications exist for the use of the other antianginal drugs (FIG. 5).

Chronic obstructive pulmonary disease. Chronic obstructive pulmonary disease is common, shares the majority of risk factors with coronary artery disease, and is the third leading cause of death ${ }^{84}$. Observational studies have found that patients with chronic obstructive pulmonary disease have a threefold increased risk of heart failure and twofold increased risk of coronary artery disease ${ }^{84}$. Historically, $\beta$-blockers are not recommended because of potential respiratory adverse effects; blocking $\beta_{2}$-adrenoceptors could lead to bronchoconstriction and worsening lung function. Evidence suggests that $\beta_{1}$-selective blockers are generally well-tolerated in patients with chronic obstructive pulmonary disease and might improve survival and, paradoxically, bronchial responsiveness ${ }^{85}$. Given its high $\beta_{1}$-selectivity, bisoprolol is the only $\beta$-blocker that is not contraindicated in chronic obstructive pulmonary disease.

Coexistence of asthma, however, is a clear contraindication to the use of $\beta$-blockers, as is chronic obstructive pulmonary disease with positive bronchoreactivity. In these patients, when treatment of chronic stable angina requires reduction of heart rate, ivabradine, diltiazem, or verapamil are preferred. For patients with chronic stable angina and preserved ventricular function, the option of calcium-channel blockers or nitrates is preferred, because $\beta$-blockers will not add any prognostic benefits. In the presence of concomitant pulmonary hypertension with right ventricular dysfunction, nondihydropyridine calcium-channel blockers and nonselective $\beta$-blockers are not recommended (FIG. 5). 
Peripheral artery disease. Intermittent claudication is the most common symptom of peripheral atherosclerotic disease, and is often associated with chronic stable angina, particularly in patients with diabetes ${ }^{86}$. Often, intermittent claudication that limits exercise time masks the occurrence of chronic stable angina. Patients with peripheral artery disease and chronic stable angina have increased mortality and, therefore, need aggressive treatment.

Traditionally, $\beta$-blockers were considered to exert general cardioprotection against ischaemia and, therefore, also to be effective in patients with concomitant peripheral artery disease. This assumption has been questioned because of presumed negative peripheral haemodynamic consequences of $\beta$-blockers, including a decrease in cardiac output and unopposed $\alpha$-adrenergic drive leading to vasoconstriction ${ }^{86,87}$. In 2013, the British Medical Association declared that $\beta$-blockers were contraindicated in severe peripheral artery disease ${ }^{88}$. The same year, a systematic Cochrane Review provided no strong evidence for or against the use of $\beta$-blockers in peripheral artery disease ${ }^{89}$. Given the paucity of reliable and contemporary data, the consensus of our group is that $\beta$-blockers should be avoided or used with caution in patients with chronic stable angina and peripheral artery disease. Equally (and especially in cases of critical ischaemia), vasodilators such as calcium-channel blockers and nitrates should also be avoided because acute blood-pressure lowering is deleterious. The remaining antianginal drugs (ivabradine, ranolazine, and trimetazidine) are preferred (FIG. 5).

Defects in atrioventricular conduction. Patients with chronic stable angina often present with atrioventricular conduction defects of different degrees. $\beta$-Blockers and nondihydropyridine calcium-channel blockers reduce atrioventricular conduction and can even cause a complete atrioventricular block and intraventricular dyssynchrony ${ }^{90}$. Therefore, a clear contraindication exists for their use in patients with second-degree atrioventricular block. To control symptoms of chronic stable angina, preference is given to the other antianginal agents (FIG. 5).

Hyperthyroidism. Hyperthyroidism is a pathological disorder in which excess thyroid hormones are synthesized and secreted by the thyroid gland. A relationship between hyperthyroidism and cardiovascular abnormality is well known. Patients with hyperthyroidism have a threefold increased risk of atrial fibrillation and consequent heart failure ${ }^{91}$. Thyroid hormone can directly affect the factors that determine oxygen consumption of the myocardium, leading to the development of chronic stable angina in patients with coronary artery disease or even with angiographically normal coronary arteries. Thyroid hormones can also cause coronary artery spasm $^{92}$. The preferred treatment in these patients is nonselective $\beta$-blockers (propanolol), or diltiazem, verapamil, or ivabradine if $\beta$-blockers are contraindicated. Vasodilators should not be used because of the risk of reflex tachycardia (FIG. 5).
Vasospastic angina. The preferred drugs to prevent and/or treat coronary artery spasm are undoubtedly calcium-channel blockers and long-acting nitrates. All calcium-channel blockers can prevent spasm in about $90 \%$ of patients. Long-acting nitrates are efficacious, but intermittent administration is important to prevent nitrate tolerance. $\beta$-Blockers are contraindicated, because they can precipitate spasm by leaving $\alpha$-mediated vasoconstriction unopposed by $\beta$-mediated vasodilatation. In patients with refractory angina, high doses of calcium-channel blockers can be tried. In the rare patients who are refractory to maximally tolerated doses of calcium-channel blockers, sympathectomy is a therapeutic option ${ }^{93}$ (FIG. 5).

Microvascular angina. No conclusive evidence is available to support a specific class of drugs, probably because of the limited knowledge on the causes of microvascular angina and the variable response to different drug treatments. For many years, traditional antianginal drugs, such as $\beta$-blockers, calcium-channel blockers, and nitrates were considered the only option, despite $20-30 \%$ of patients remaining symptomatic. $\beta$-Blockers might be preferred in patients with evidence of increased adrenergic activity ${ }^{1}$. Ranolazine has been suggested to reduce mechanical compression of coronary microcirculation ${ }^{94,95}$. In addition, ranolazine might also improve coronary self-regulation ${ }^{96}$. A small trial with ranolazine suggested improvement of anginal symptoms in women with microvascular angina ${ }^{97}$. Subsequently, in a double-blind, placebo-controlled, crossover study conducted in women with effort angina but no obstructive coronary artery disease, ranolazine produced no significant benefits except in some patients with impaired coronary flow reserve ${ }^{65}$. Analogously, ivabradine has been shown to improve coronary collateral flow and coronary flow reserve in patients with microvascular angina ${ }^{47,48}$. The effects of ivabradine were superior to those of bisoprolol, despite similar degrees of heart-rate reduction ${ }^{48}$. Therefore, treatment of microvascular angina is extremely challenging and necessarily empirical. Slowing the heart rate with $\beta$-blockers, diltiazem, verapamil, or ivabradine, can be considered because of increased diastolic time and coronary perfusion. Co-administration of ranolazine or trimetazidine might also be useful. In patients with enhanced pain perception, adenosine antagonists and drugs effective in chronic pain syndromes, such as imipramine (a tricyclic antidepressant), are other therapeutic options.

\section{Conclusions}

Given that all antianginal drugs have similar efficacy and level of evidence, and no survival benefit, considering some drugs but not others to be 'first choice' is difficult. Furthermore, patients with stable ischaemic heart disease and chronic stable angina can have several comorbidities, and cardiac ischaemic pain might result from various underlying pathophysiologies. Some agents, in addition to their antianginal effect, have auxiliary properties that could be useful, depending on comorbidities and the mechanisms of the chronic stable angina. 
During our discussion, we reached a consensus that the time has come to propose a different, more individualized approach to patients, their comorbidities, and mechanisms of chronic stable angina: the 'diamond' approach. We recognize that drug management of patients with chronic stable angina is challenging, particularly in those with chronic stable angina and normal coronary arteries, a condition in which considerable work remains to be done to address optimal therapy definitively. We are also aware that the issue of reperfusion has not been discussed, and that readers might be left with many unanswered questions, but we hope that our approach will help clinicians to make the best possible therapeutic choices, independently of whether the drugs are first or second choice.
1. Task Force Members et al. 2013 ESC guidelines on the management of stable coronary artery disease: the Task Force on the management of stable coronary artery disease of the European Society of Cardiology. Eur. Heart J. 34, 2949-3003 (2013).

2. Finn, S. D. et al. 2012 ACCF/AHA/ACP/AATS/PCNA/ SCAI/STS guideline for the diagnosis and management of patients with stable ischemic heart disease: a report of the American College of Cardiology Foundation/ American Heart Association Task Force on Practice Guidelines, and the American College of Physicians, American Association for Thoracic Surgery, Preventive Cardiovascular Nurses Association, Society for Cardiovascular Angiography and Interventions, and Society of Thoracic Surgeons. J. Am. Coll. Cardiol. 60 e44-e164 (2012).

3. Mancini, G. B. et al. Canadian Cardiovascular Society guidelines for the diagnosis and management of stable ischemic heart disease. Can. J. Cardiol. 30, 837-849 (2014).

4. National Clinical Guidelines Centre. Stable angina: methods, evidence \& guidance. NICE http:// www.nice.org.uk/guidance/cg126/evidence/ full-guideline-183176605 (2011)

5. Thadani, U. Management of stable angina - current guide lines: a critical appraisal. Cardiovasc. Drugs Ther. 30, 419-426 (2016).

6. Camm, A. J. et al. Unresolved issues in the management of chronic stable angina. Int. J. Cardiol. 201, 200-207 (2015).

7. Ambrosio, G. et al. Management of stable angina: a commentary on the European Society of Cardiology guidelines. Eur. J. Prev. Cardiol. 23, 1401-1412 (2016).

8. Manolis, A. J. et al. Medical treatment of stable angina: a tailored therapeutic approach. Int. J. Cardiol. 220, 445-453 (2016).

9. Marzilli, M. et al. Obstructive coronary atherosclerosis and ischemic heart disease: an elusive link! J. Am. Coll. Cardiol. 60, 951-956 (2012).

10. Crea, F., Camici, P. G. \& Bairey Merz, C. N. Coronary microvascular dysfunction: an update. Eur. Heart J. 35, 1101-1111 (2014)

11. Stergiopoulos, K. et al. Percutaneous coronary intervention outcomes in patients with stable obstructive coronary artery disease and myocardial ischemia: a collaborative meta-analysis of contemporary randomized clinical trials. JAMA Intern. Med. 174, 232-240 (2014)

12. McGill, H. C. Jr et al. Association of coronary heart disease risk factors with microscopic qualities of coronary atherosclerosis in youth. Circulation 102 374-379 (2000)

13. Vermeltfoort, I. A. et al. Definitions and incidence of cardiac syndrome $\mathrm{X}$ : review and analysis of clinical data. Clin. Res. Cardiol. 99, 475-481 (2010).

14. Paul, T. K., Sivanesan, K. \& Schulman-Marcus, J. Sex differences in non obstructive coronary artery disease: recent insights and substantial knowledge gaps. Trends Cardiovasc. Med. 27, 173-179 (2016)

15. Marinescu, M. A. et al. Coronary microvascular dysfunction, microvascular angina, and treatment strategies. JACC Cardiovasc. Imaging 8, 210-220 (2015).

16. Lanza, G. A. \& Crea, F. Primary coronary microvascular dysfunction: clinical presentation pathophysiology and management. Circulation 121, 2317-2325 (2010)

17. Sedlak, T., Izadnegahdar, M., Humphries, K. H. \& Bairey Merz, C. N. Sex-specific factors in microvascular angina. Can. J. Cardiol. 30, 747-755 (2014)

18. Maseri, A. Coronary artery spasm and thrombosis. Cardiovascular Clinics, vol 14 No. 1. J. R. Soc. Med. 78, 178 (1985)

19. Chrysant, S. G. et al. Efficacy and safety of extendedrelease isosorbide mononitrate for stable effort angina pectoris. Am. J. Cardiol. 72, 1249-1256 (1993).
20. O'Rourke, S. T. Nitro vasodilators: pharmacology and use in the treatment of myocardial ischemia. Am. J. Pharm. Educ. 66, 177-180 (2002)

21. Thadani, U. \& Rodgers, T. Side effects of using nitrates to treat angina. Expert Opin. Drug Saf. 5, 667-674 (2006)

22. Heidenreich, P. A. et al. Meta-analysis of trials comparing beta-blockers, calcium antagonists, and nitrates for stable angina. JAMA 281, 1927-1936 (1999).

23. Belsey, J., Savelieva, I., Mugelli, A. \& Camm, A. J Relative efficacy of antianginal drugs used as add-on therapy in patients with stable angina: a systematic review and meta-analysis. Eur. J. Prev. Cardiol. 22, 837-848 (2015).

24. Salazar, C. A. et al. Ranolazine for stable angina pectoris. Cochrane Database Syst. Rev. 2, CD011747 (2017).

25. Thadani, U. \& Opie, L. H. in Drugs for the Heart 4th edn (ed. Opie, L. H.) 31-48 (Saunders, 1995).

26. Daiber, A. \& Münzel, T. Organic nitrate therapy, nitrate tolerance, and nitrate-induced endothelial dysfunction: emphasis on redox biology and oxidative stress. Antioxid. Redox Signal. 23, 899-942 (2015).

27. Husted, S. E. \& Ohman, E. M. Pharmacological and emerging therapies in the treatment of chronic angina. Lancet 386, 691-701 (2015)

28. Thadani, U. et al. Comparison of the immediate effects of five beta adrenoreceptor-blocking drugs with different ancillary properties in angina pectoris. N. Engl. J. Med. 300, 750-755 (1979).

29. Tardif, J. C. et al. Efficacy of the $\mathrm{I}_{\mathrm{f}}$ current inhibitor ivabradine in patients with chronic stable angina receiving beta-blocker therapy: a 4-month, randomized, placebo-controlled trial. Eur. Heart J. 30 540-548 (2009)

30. Bangalore, S. et al. Clinical outcomes with betablockers for myocardial infarction: a meta-analysis of randomized trials. Am. J. Med. 127, 939-953 (2014).

31. Bangalore, S. et al. $\beta$-blocker use and clinical outcomes in stable outpatients with and without coronary artery disease. JAMA 308, 1340-1349 (2012).

32. Ferrari, R. Coronary artery disease in 2012: revising common beliefs in the management of stable CAD. Nat. Rev. Cardiol. 10, 65-66 (2013).

33. Ferrari, R. \& Fox, K. Heart rate reduction in coronary artery disease and heart failure Nat. Rev. Cardiol. 13 , 493-501 (2016)

34. Andersson, C. et al. $\beta$-blocker therapy and cardiac events among patients with newly diagnosed coronary heart disease. J. Am. Coll. Cardiol. 64, 247-252 (2014).

35. Booij, H. G. et al. $\beta$-blocker therapy is not associated with reductions in angina or cardiovascular events after coronary artery bypass graft surgery: insights from the IMAGINE trial. Cardiovasc. Drugs Ther. 29 277-285 (2015)

36. Antman, E. et al. Nifedipine therapy for coronaryartery spasm: experience in 127 patients. N. Engl. J. Med. 302, 1269-1273 (1980).

37. The Multicenter Diltiazem Postinfarction Trial Research Group. The effect of diltiazem on mortality and reinfarction after myocardial infarction. N. Engl. J. Med. 319, 385-392 (1988)

38. Poole-Wilson, P. A. et al. Effect of long-acting nifedipine on mortality and cardiovascular morbidity in patients with stable angina requiring treatment (ACTION trial): randomised controlled trial. Lancet 364, 849-857 (2004).

39. Fox, K. et al. Ivabradine in stable coronary artery disease without clinical heart failure. N. Engl. J. Med. 371, 1091-1099 (2014)

40. Suryapranata, H. et al. Coronary vasodilatory action after a single dose of nicorandil. Am. J. Cardiol. 61, 292-297 (1988).
41. Gupta, A. K., Winchester, D. \& Pepine, C. J. Antagonist molecules in the treatment of angina. Expert Opin. Pharmacother. 14, 2323-2342 (2013)

42. Camm, A. J. \& Maltz, M. B. A controlled single-dose study of the efficacy, dose response and duration of action of nicorandil in angina pectoris. Am. J. Cardiol. 63, 61J-65J (1989).

43. Thadani, U. Can nicorandil treat angina pectoris effectively? Nat. Clin. Pract. Cardiovasc. Med. 2, 186-187 (2005).

44. The IONA Study Group. Effect of nicorandil on coronary events in patients with stable angina: the Impact Of Nicorandil in Angina (IONA) randomised trial. Lancet 359, 1269-1275 (2002).

45. Borer, J. S. \& Heuzey, J. Y. Characterization of the heart rate-lowering action of ivabradine, a selective $\mathrm{I}_{\mathrm{t}}$ current inhibitor. Am. J. Ther. 15, 461-473 (2008).

46. Simon, L. et al. Coronary and hemodynamic effects of S 16257, a new bradycardic agent, in resting and exercising conscious dogs. J. Pharmacol. Exp. Ther. 275, 659-666 (1995).

47. Gloekler, S. et al. The effect of heart rate reduction by ivabradine on collateral function in patients with chronic stable coronary artery disease. Heart 100, 160-166 (2014)

48. Tagliamonte, E. et al. Ivabradine and bisoprolol on doppler-derived coronary flow velocity reserve in patients with stable coronary artery disease: beyond the heart rate. Adv. Ther. 32, 757-767 (2015).

49. Werdan, $\mathrm{K}$ et al. Effectiveness of ivabradine treatment in different subpopulations with stable angina in clinical practice: a pooled analysis of observational studies. Cardiology 135, 141-150 (2016).

50. Swedberg, K et al. Ivabradine and outcomes in chronic heart failure (SHIFT): a randomised placebocontrolled study. Lancet 376, 875-885 (2010).

51. Fox, K. et al. Bradycardia and atrial fibrillation in patients with stable coronary artery disease treated with ivabradine: an analysis from the SIGNIFY study. Eur. Heart J. 36, 3291-3296 (2015).

52. Tardif, J. C. et al. Efficacy of ivabradine, a new selective $\mathrm{I}_{\mathrm{f}}$ inhibitor, compared with atenolol in patients with chronic stable angina. Eur. Heart J. 26, 2529-2536 (2005).

53. Ruzyllo, W., Tendera, M., Ford, I. \& Fox, K. M Antianginal efficacy and safety of ivabradine compared with amlodipine in patients with stable effort angina pectoris: a 3-month randomised, double-blind, multicentre, noninferiority trial. Drugs 67, 393-405 (2007).

54. Amosova, E. et al. Efficacy of ivabradine in combination with beta-blocker versus uptitration of beta-blocker in patients with stable angina. Cardiovasc. Drugs Ther. 25, 531-537 (2011).

55. European Medicines Agency: Pharmacovigilance Risk Assessment Committee (PRAC). Assessment report: ivabradine. EMA http://www.ema.europa.eu/docs/en GB/document library/EPAR - Assessment Report Variation/human/000597/WC500182140.pdf (2014).

56. Fox, K. et al. Ivabradine for patients with stable coronary artery disease and left-ventricular systolic dysfunction (BEAUTIFUL): a randomised, double-blind, placebo-controlled trial. Lancet 372, 807-816 (2008).

57. Fox, K. et al. Heart rate as a prognostic risk factor in patients with coronary artery disease and left-ventricular systolic dysfunction (BEAUTIFUL): a subgroup analysis of a randomised controlled trial. Lancet 372, 817-821 (2008).

58. Fox, K. et al. Relationship between ivabradine treatment and cardiovascular outcomes in patients with stable coronary artery disease and left ventricular systolic dysfunction with limiting angina: a subgroup analysis of the randomized, controlled BEAUTIFUL trial. Eur. Heart J. 30, 2337-2345 (2009). 
59. Borer, J. S. et al. Efficacy profile of ivabradine in patients with heart failure plus angina pectoris. Cardiology 136, 138-144 (2016).

60. Rayner-Hartley, E. \& Sedlak, T. Ranolazine: a contemporary review. J. Am. Heart Assoc. 5, e003196 (2016)

61. McCormack, J. G., Barr, R. L., Wolff, A. A. \& Lopaschuk, G. D. Ranolazine stimulates glucose oxidation in normoxic, ischemic, and reperfused ischemic rat hearts. Circulation 93, 135-142 (1996).

62. Thadani, U., Ezekowitz, M., Fenney, L. \& Chiang, Y. K. Double-blind efficacy and safety study of a novel antiischemic agent, ranolazine, versus placebo in patients with chronic stable angina pectoris. Circulation 90 , 726-734 (1994)

63. Weisz, G. et al. Use of ranolazine in patients with incomplete revascularization after percutaneous coronary intervention: design and rationale of the Ranolazine for Incomplete Vessel Revascularization Post-Percutaneous Coronary Intervention (RIVER-PCI) trial. Am. Heart J. 166, 953.e3-959.e3 (2013).

64. Morrow, D. A. et al. Effects of ranolazine on recurrent cardiovascular events in patients with non-ST-elevation acute coronary syndromes: the MERLIN-TIMI 36 randomized trial. JAMA 297, 1775-1783 (2007).

65. Bairey Merz, C. N. et al. A randomized, placebocontrolled trial of late $\mathrm{Na}$ current inhibition (ranolazine) in coronary microvascular dysfunction (CMD): impact on angina and myocardial perfusion reserve. Eur. Heart J. 37, 1504-1513 (2016).

66. Kantor, P. F., Lucien, A., Kozak, R. \& Lopaschuk, G. D. The antianginal drug trimetazidine shifts cardiac energy metabolism from fatty acid oxidation to glucose oxidation by inhibiting mitochondrial longchain 3-ketoacyl coenzyme A thiolase. Circ. Res. 86 580-588 (2000).

67. Fragasso, G. et al. Effects of metabolic modulation by trimetazidine on left ventricular function and phosphocreatine/adenosine triphosphate ratio in patients with heart failure. Eur. Heart J. 27, 942-948 (2006).

68. McClellan, K. J. \& Plosker, G. L. Trimetazidine. A review of its use in stable angina pectoris and other coronary conditions. Drugs 58, 143-157 (1999)

69. Detry, J. M. et al. Trimetazidine: a new concept in the treatment of angina. Comparison with propranolol in patients with stable angina. Br. J. Clin. Pharmacol. 37, 279-288 (1994)

70. Peng, S. et al. The efficacy of trimetazidine on stable angina pectoris: a meta-analysis of randomized clinical trials. Int. J. Cardiol. 177, 780-785 (2014).

71. Xu, X. et al. Effect of trimetazidine on recurrent angina pectoris and left ventricular structure in elderly multivessel coronary heart disease patients with diabetes mellitus after drug-eluting stent implantation: a single-centre, prospective, randomized, double-blind study at 2-year follow-up. Clin. Drug Investig. 34, 251-258 (2014)

72. Vidal-Petiot, E. et al. Cardiovascular event rates and mortality according to achieved systolic and diastolic blood pressure in patients with stable coronary artery disease: an international cohort study. Lancet 388 , 2142-2152 (2016)

73. Tsika, E. P., Poolimenos, L. E., Boudoulas, K. A \& Manolis, A. I. The J curve in arterial hypertension: fact or fallacy? Cardiology 129, 126-135 (2014).

74. Ponikowski, P. et al. 2016 ESC guidelines for the diagnosis and treatment of acute and chronic heart failure: the Task Force for the diagnosis and treatment of acute and chronic heart failure of the European Society of Cardiology (ESC). Developed with the specia contribution of the Heart Failure Association (HFA) of the ESC. Eur. J. Heart Fail. 18, 891-975 (2016)

75. Gao, D., Ning, N., Niu, X., Hao, G. \& Meng, Z. Trimetazidine: a meta-analysis of randomised controlled trials in heart failure. Heart 97, 278-286 (2011).
76. Martin, R. I. et al. Atrial fibrillation associated with ivabradine treatment: meta-analysis of randomised controlled trials. Heart 100, 1506-1510 (2014).

77. Scirica, B. M. et al. Effect of ranolazine, an antianginal agent with novel electrophysiological properties, on the incidence of arrhythmias in patients with non ST-segment elevation acute coronary syndrome: results from the Metabolic Efficiency with Ranolazine for Less Ischemia in Non ST-Elevation Acute Coronary Syndrome Thrombolysis in Myocardial Infarction 36 (MERLIN-TIMI 36) randomized controlled trial. Circulation 116, 1647-1652 (2007).

78. Reiffel, J. A. et al. The HARMONY trial: combined ranolazine and dronedarone in the management of paroxysmal atrial fibrillation: mechanistic and therapeutic synergism. Circ. Arrhythm. Electrophysiol. 8, 1048-1056 (2015)

79. Danchin, N., Marzilli, M., Parkhomenko, A \& Ribeiro, J. P. Efficacy comparison of trimetazidine with therapeutic alternatives in stable angina pectoris: a network meta-analysis. Cardiology 120, 59-72 (2011)

80. Timmis, A. D., Chaitman, B. R. \& Crager, M. Effects of ranolazine on exercise tolerance and $\mathrm{HbA} 1 \mathrm{c}$ in patients with chronic angina and diabetes. Eur. Heart J. 27, 42-48 (2006).

81. Kosiborod, M. et al. Evaluation of ranolazine in patients with type 2 diabetes mellitus and chronic stable angina: results from the TERISA randomized clinical trial (type 2 diabetes evaluation of ranolazine in subjects with chronic stable angina). J. Am. Coll. Cardiol. 61, 2038-2045 (2013).

82. Elliott, W. J. \& Meyer, P. M. Incident diabetes in clinical trials of antihypertensive drugs: a network meta-analysis. Lancet 369, 201-207 (2007).

83. Jerling, M. Clinical pharmacokinetics of ranolazine. Clin. Pharmacokinet. 45, 469-491 (2006).

84. Schnell, K. et al. The prevalence of clinically relevant comorbid conditions in patients with physiciandiagnosed COPD: a cross-sectional study using data from NHANES 1999-2008. BMC Pulm. Med. 12, 26 (2012)

85. Rutten, F. H. et al. Beta-blockers may reduce mortality and risk of exacerbations in patients with chronic obstructive pulmonary disease. Arch. Intern. Med. 170, 880-887 (2010)

86. Hiatt, W. R., Stoll, S. \& Nies, A. S. Effect of betaadrenergic blockers on the peripheral circulation in patients with peripheral vascular disease. Circulation 72, 1226-1231 (1985)

87. George, C. F. Beta-receptor blocking agents Prescribers J. 14, 93-98 (1974).

88. Joint Formulary Committee. British National Formulary Vol. 65 (Pharmaceutical Press, 2013).

89. Paravastu, S. C., Mendonca, D. A. \& Da Silva, A Beta blockers for peripheral arterial disease. Cochrane Database Syst. Rev. 9, CD005508 (2013)

90. Miller, J. M. \& Zipes, D. P. in Heart Disease A Textbook of Cardiovascular Medicine 6th edn (eds Braunwald, E., Zipes, D. P. \& Libby, P.) 711-739 (Saunders, 2001).

91. De Leo, S., Lee, S. Y. \& Braverman, L. E. Hyperthyroidism. Lancet 388, 906-918 (2016)

92. Masini, N. D., Northridge, D. B. \& Hall, R. J. C. Severe coronary vasospasm associated with hyperthyroidism causing myocardial infarction. Br. Heart J. 74 700-701 (1995).

93. Abbate, A. Sympathectomy as a treatment for refractory coronary artery spasm. Int J. Cardiol. 161 e7-e9 (2012).

94. Cattaneo, M., Poretta, A. P. \& Gallino, A. Ranolazine: drug overview and possible role in primary microvascular angina management. Int. J. Cardiol. 181, 376-381 (2015)

95. Venkataraman, R., Belardinelli, L., Blackburn, B., Heo, J. \& Iskandrian, A. E. A study of the effects of ranolazine using automated quantitative analysis of serial myocardial perfusion images. JACC CardiovasC. Imaging 2, 1301-1309 (2009).

96. Tagliamonte, E. et al. Effects of ranolazine on noninvasive coronary flow reserve in patients with myocardial ischemia but without obstructive coronary artery disease. Echocardiography 32, 516-521 (2015).

97. Mehta, P. K. et al. Ranolazine improves angina in women with evidence of myocardial ischemia but no obstructive coronary artery disease. JACC CardiovasC. Imaging 4, 514-522 (2011).

98. Maddox, T. M. et al. Angina at 1 year after myocardial infarction: prevalence and associated findings. Arch. Intern. Med. 168, 1310-1316 (2008).

99. National Institutes of Health/National Heart, Lung, and Blood Institute. Morbidity \& Mortality: 2012 Chart Book on Cardiovascular, Lung, Blood Diseases. National Heart, Lung and Blood Institute https://www.nhlbi.nih.gov/files/docs/research/ 2012 ChartBook 508.pdf (2012).

\section{Acknowledgements}

This Consensus Statement originated from the University of Ferrara through a meeting supported by a grant from Fondazione Anna Maria Sechi per il Cuore (FASC), Italy, a generous bequest on behalf of Antonio Filippini, and an unrestricted educational grant from Servier International. FASC and Servier had no role in the preparation of the manuscript or the decision to publish.

\section{Author contributions}

All authors contributed to researching data, discussion of content, and reviewing and editing the manuscript before submission. R.F. wrote the manuscript.

\section{Competing interests statement}

R.F. has received honoraria for steering committee membership and consulting from Novartis and Servier; and for speaking and support for travel to study meetings from Amgen, Bayer, Boehringer Ingelheim, Merck Serono, and Servier. P.G.C. is a consultant for Servier, is part of Board meetings of AstraZeneca, and has received speaking honoraria from Menarini and Servier. F.C. has received honoraria for speaking from BMS, Menarini, Novartis, Sanofi, and Servier; and received grants from Biotronik and Boeringher Ingelheim. K.F. has received personal fees, honoraria, and/or travel expenses from Armgo, AstraZeneca, Broadview Ventures, CellAegis, Servier, and TaurX; and is a director of Vesalius Trials Ltd. A.P.M. has received honoraria for steering committee membership from Bayer, Cardiorentis, and Novartis; received support for travel to study meetings from the same companies; and received personal fees for speaking activities from Amgen, Lilly, Sanofi, and Servier. J.L.L.-S. has received honoraria for steering committee membership from AstraZeneca, Bayer, Boehringer-Ingelheim, GlaxoSmithKline, Menarini, Merck, Novartis, Pfizer, Sanofi, and Servier; received honoraria for speaking from Amgen and Sanofi; and received honoraria for consultancy from Boehringer Ingelheim and Menarini. The other authors declare no competing interests.

\section{Publisher's note}

Springer Nature remains neutral with regard to jurisdictional claims in published maps and institutional affiliations.

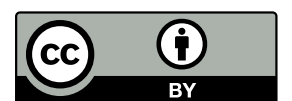

This work is licensed under a Creative Commons Attribution 4.0 International License. The images or other third party material in this article are included in the article's Creative Commons license, unless indicated otherwise in the credit line; if the material is not included under the Creative Commons license, users will need to obtain permission from the license holder to reproduce the material. To view a copy of this license, visit http://creativecommons.org/licenses/by/4.0/. 